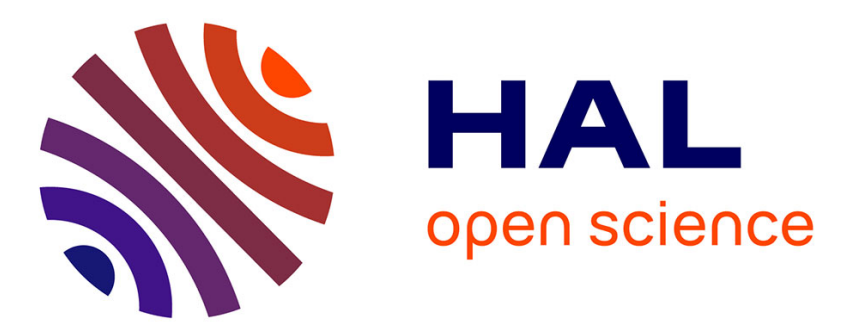

\title{
Work of indentation approach to the analysis of hardness and modulus of thin coatings
}

Alexander Korsunsky, Andreï Constantinescu

\section{To cite this version:}

Alexander Korsunsky, Andreï Constantinescu. Work of indentation approach to the analysis of hardness and modulus of thin coatings. Materials Science and Engineering: A, 2006, 423, pp.28-35. 10.1016/j.msea.2005.09.126 . hal-00111507

\section{HAL Id: hal-00111507 \\ https://hal.science/hal-00111507}

Submitted on 24 Jul 2019

HAL is a multi-disciplinary open access archive for the deposit and dissemination of scientific research documents, whether they are published or not. The documents may come from teaching and research institutions in France or abroad, or from public or private research centers.
L'archive ouverte pluridisciplinaire HAL, est destinée au dépôt et à la diffusion de documents scientifiques de niveau recherche, publiés ou non, émanant des établissements d'enseignement et de recherche français ou étrangers, des laboratoires publics ou privés. 


\title{
Work of indentation approach to the analysis of hardness and modulus of thin coatings
}

\author{
Alexander M. Korsunsky ${ }^{\mathrm{a}, *}$, Andrei Constantinescu ${ }^{\mathrm{b}}$ \\ ${ }^{a}$ Department of Engineering Science, University of Oxford, Parks Road, Oxford OX1 3PJ, United Kingdom \\ ${ }^{\mathrm{b}}$ Laboratoire de Mecanique des Solides, CNRS UMR 7649, Ecole Polytechnique, 91128 Palaiseau cedex, France
}

\begin{abstract}
The problem of accurate identification of mechanical properties, primarily hardness and modulus, invariably arises whenever very thin coatings are considered that consist of single or multiple layers, e.g. in the context of fitness-for-purpose characterisation of coated systems, monitoring in-service degradation, component lifetime prediction, etc. Rapid developments in the areas of nano-fabrication, nano-manipulation and nanotechnology lead to the increased importance of reliable characterisation of mechanical properties of progressively thinner coatings. Instrumented small scale (nano-) indentation is particularly well-suited to surface-engineered and thin-coated systems. The present study presents a review and refinement of the interpretation techniques for instrumented (nano) indentation for reliable property de-convolution of coated systems. Particular attention is devoted to contact modulus and hardness, as the properties that play the key role in controlling the deformation response of any surface, and affect such service properties as impact and erosion resistance, wear and fretting fatigue resistance, resistance to crack initiation and propagation, etc. A flexible multi-scaling power law functional description is introduced and discussed, and its application to various example systems is illustrated.
\end{abstract}

Keywords: Contact; Modulus; Hardness; Coating; Property identification

\section{Introduction}

\subsection{Motivation}

In many situations of industrial manufacture one of the key problems is that of achieving optimal service properties of engineering components. Often these problems are best solved by using surface engineering techniques, e.g. physico-chemical surface treatment, or deposition of thin coating layers over the existing substrate. This approach minimises the expense of using high performance materials, such as very hard diamond-like carbon films for cutting tool applications, or highly heat-resistant yttria-stabilized zirconia ceramics for thermal barrier coating on aeroengine turbine blades. It also often provides the best balance between the properties of the coating (hardness, low thermal conductivity) and those of the substrate (e.g. the ductility and toughness of a load bearing metallic alloys).

\footnotetext{
* Corresponding author. Tel.: +44 1865 273043; fax: +44 1865273010.

E-mail address: alexander.korsunsky@eng.ox.ac.uk (A.M. Korsunsky).
}

In many such situations the problem of property identification arises for the thin single or multiple coating layers. Initially the requirement may arise in the context of fitness-for-purpose characterisation of the coating-substrate system, which is followed by the need to carry out process optimisation in order to achieve the best performance. Finally, property identification is important in the context of monitoring the in-service degradation, and for predicting component lifetime reliably.

The conventional approach to the task of system characterisation often relies on full or partial simulation of the in-service conditions. With this purpose in mind large scale tests of equipment are performed, often to destruction. Clearly experiments of this kind are extremely expensive and very time-consuming. Scaled-down laboratory rigs, such as wear testers, are very useful in capturing some aspects of the process. However, they often require preparation of specimens from small coupons, and thus cannot be considered as non-destructive.

The alternative to these approaches that is particularly wellsuited to surface-engineered and coated systems is small scale surface indentation. When an indenter made from a hard material such as diamond is brought into contact with the sample surface, 
the contact initially is purely elastic. This is reflected in the same load-displacement trace being repeatedly passed during unloading. The information contained in the load-displacement curve in principle allows the contact modulus of the coating alone to be determined, provided the range of loads considered is so small that the substrate deformation can be disregarded. However, in practice the measurement errors for displacement and load become progressively larger as the contact load is reduced, making accurate determination of mechanical properties of the coating difficult.

Considering the importance of reliable characterisation requirement for progressively thinner coatings finding widespread use in industry, this study aims to review and refine the techniques using instrumented indentation for reliable property de-convolution of coated systems. We focus our attention on two properties of most relevance to the mechanical performance of coated systems, namely, contact modulus and hardness. These two parameters play a key role in controlling the deformation response of any surface, and affect such service properties as impact and erosion resistance, wear and fretting fatigue resistance, resistance to crack initiation and propagation, etc.

\subsection{Article layout}

This paper reviews the construction and application of an approach that aims to identify the underlying relationship between the observed contact response (displacement of the indenter tip under given applied load), the coating thickness and the mechanical properties of the substrate/coating combination. The purpose of the study is to provide fast and efficient means of extracting material property data from indentation tests.

When a suitably sharp indenter is used (i.e. the tip radius of curvature is several orders of magnitude smaller than the contact radius and the coating thickness), then the response of the system may be assumed dominated by inelastic processes, such as fracture or plasticity. In this case detailed modelling of the elasto-plastic deformation process is required to predict the load-displacement curve. However, as a first step we present a qualitative analysis based on the work of indentation that allows some predictions to be made. In particular, it is possible to use the work of indentation approach to identify the functional form of the dependence of the apparent system hardness on the indentation depth.

The function identified in this way is termed the indentation response function. It is used extensively to interpret a series of displacement-dependent hardness measurements in coated systems, The reliability of the fit to experimental data that is obtained using the indentation response function provides some confidence in its utility. In order to provide further confirmation for the use of the indentation response function its predictions are compared with both experimental measurements and the result of elasto-plastic finite element modelling of the indentation process.

It is interesting to pose a question about the cases when purely elastic response is observed. In such cases the solution is not sensitive to the peculiarities of the material non-linear response. In the present paper, a semi-analytical model is applied, based on the axially symmetric integral equation relating the stress distribution within the contact area, on the one hand, and the vertical displacement on the other. The model is implemented in a highly efficient numerical procedure, and the results used to formulate a special indentation response function for the coating/substrate composite. The function parameters can be tuned up to the observed response, and the ultimate coating-only properties be extracted in an easy way. In fact, the response to any applied load can then be routinely predicted.

It is remarkable that the indentation response function suitable for describing the hardness variation can also be used for predicting the apparent elastic contact modulus. The principal utility of this function is in the ability to de-convolve the unknown properties without invoking complex and computationally expensive inverse method procedures.

\section{Hardness response}

\subsection{Background}

Understanding the contact response of coated systems to the point whereby reliable quantitative models can be constructed both to explain and predict performance is a critical step in the selection and optimisation of coatings for particular substrates and applications. However, since the mechanical response of coated systems will vary with contact severity and scale, it is important to develop soundly-based models that allow the performance over a suitably wide range of scales to be successfully predicted. In this context, there is general agreement that at contact scales of dimensions less than the coating thickness $(t)$, the coating dominates the system response, while at scales which are very large compared to $t$, the substrate dominates with a mixed response occurring at intermediate scales. However, many previous attempts to model this behaviour quantitatively have largely failed, generally because they encountered one of the two problems. The first has been the difficulty of obtaining good experimental data at contact scales less than the coating thickness - something necessary to enable models to be both constructed and tested over the necessary wide range of scales involved. The second problem has been the selection of an appropriate model that can be applied at all contact scales. Previous models have been of two types, system models which separate the measured contact response into contributions from coating and substrate without detailed treatment of the deformation mechanisms in each [2-12], and mechanistic models which consider the effect of a given deformation mechanism on the measured contact response [13-15]. System models become invalidated when major changes in deformation occur (e.g. cracking of coatings) that conflict with assumptions on which the model is based. Similarly, mechanistic modes have significant shortcomings when used to fit experimental data originating from systems displaying other modes of deformation. In general, most coated systems show mixtures of deformation modes and thus there is a clear need for a model which, while having soundly-based physical origins, is capable of dealing with such generalised responses. There is a further need for any such model to be sufficiently mathematically 
tractable as to allow straightforward fitting procedures to be used.

In the present overview we only provide short references to some of the most significant previous attempts at describing the contact response of coated systems. For a more detailed discussion of the historical aspect of the problem the reader is referred to the earlier paper (Korsunsky et al. [1]). Buckle [2] thought of the observed hardness as the sum of the varying hardness values within the plastic zone (or "influence zone") at various depths beneath the indentation multiplied by some weighting factor. The model could explain work-hardening effects, but critically depended on the choice of weighting factors. Using area law-of-mixtures approach, Jonsson and Hogmark [3] expressed composite hardness $H_{\mathrm{c}}$ as follows:

$H_{\mathrm{c}}=\frac{A_{\mathrm{f}}}{A} H_{\mathrm{f}}+\frac{A_{\mathrm{s}}}{A} H_{\mathrm{s}}$

where $A_{\mathrm{f}}$ and $A_{\mathrm{s}}$ are the load-supporting areas of the film and the substrate, respectively, $A$ the total projected contact area $\left(A=A_{\mathrm{S}}+A_{\mathrm{F}}\right)$ and $H_{\mathrm{f}}$ and $H_{\mathrm{S}}$ are the hardness values for the coating and substrate. Coating fracture in response to indentation is implied, i.e. that load support in the coating arises from unfractured material at the rim of the contact. Geometric consideration of the rim size led Johnsson and Hogmark to express composite hardness as

$H_{\mathrm{c}}=H_{\mathrm{s}}+\left[2 c\left(\frac{t}{d}\right)-c^{2}\left(\frac{t}{d}\right)^{2}\right]\left(H_{\mathrm{f}}-H_{\mathrm{s}}\right)$

where $t$ is the coating thickness, $d$ the indentation diagonal, and $c$ is a constant dependent on the indenter geometry. The model does not capture the indentation size effect (ISE) at small sizes, although ISE behaviour was later included in the form

$H(d)=H_{0}+\frac{k}{d}$

where $k$ is a constant and $H_{0}$ is the hardness at very large loads was subsequently added to increase the applicability of the model [4].

The volume law-of-mixtures model originally suggested by Sargent [5] assumed plastic deformation response of both coating and substrate, and was subsequently extended variously by Burnett, Page, Rickerby and Bull [6-9]. Deforming volumes within coating and substrate, $V_{\mathrm{f}}$ and $V_{\mathrm{s}}$, respectively, contribute to composite hardness (for $H_{\mathrm{f}}>H_{\mathrm{s}}$ ) as

$H_{\mathrm{c}}=\frac{V_{\mathrm{f}}}{V} H_{\mathrm{f}}+\frac{V_{\mathrm{s}}}{V} \chi^{3} H_{\mathrm{s}}$

where $V$ is total volume $\left(V=V_{\mathrm{f}}+\chi^{3} V_{\mathrm{s}}\right)$ and $H_{\mathrm{f}}$ and $H_{\mathrm{s}}$ both include ISE behaviour of the form

$H(d)=H_{1} d^{n-2}$

where $H_{1}$ and $n$ are the constants, $H_{1}$ a hardness value at standard indentation size, and $n$ is the ISE index (equal to 2 if no ISE occurs) [10]. Here $\chi$ is a dimensionless factor which represents the modification to the deforming volume of the soft substrate due to the constraint caused by the presence of the coating. This is expected to be a strong function of the difference between the plastic zone radii in the coating and substrate. Based on an expression due to Lawn et al. [11], Burnett and Rickerby expected $\chi$ to take the form of a power law

$\chi=\left(\frac{E_{\mathrm{f}} H_{\mathrm{s}}}{E_{\mathrm{s}} H_{\mathrm{f}}}\right)^{m}$

where $E_{\mathrm{f}}$ and $E_{\mathrm{s}}$ are the Young's moduli of coating and substrate, $H_{\mathrm{f}}$ and $H_{\mathrm{s}}$ the hardness values of coating and substrate at the scale of the contact (i.e. incorporating any indentation size effects) and $m$ is a constant which can be determined by fitting to experimental data. This approach is successful at separating hardness of coating from composite hardness when plasticity dominates, i.e. there is little or no fracture.

Thomas [12] attempted to use the fact that empirical data for $H_{\mathrm{c}}$ often fits the form

$H_{\mathrm{c}}=A+\frac{B}{d}$

where $A$ and $B$ are constants, similarly to the form of indentation size effect equation used by Vingsboo et al., Eq. (3), and effectively treats the coating as a contribution to the ISE. Despite dubious physical basis this approach gives a convenient and simple formula

$H_{\mathrm{f}}=A+\left(\frac{B_{\mathrm{c}}-B_{\mathrm{s}}}{t}\right)$

where $B_{\mathrm{c}}$ and $B_{\mathrm{s}}$ are the constants measured for composite and substrate, respectively and $A$ is the large-depth hardness (i.e. of the substrate). Note that Eq. (8) only provides a value for $H_{\mathrm{f}}$, rather than describing the system response over a range of scales, that is critically dependent on the empirical constant $B_{\mathrm{c}}$. McGurk and co-workers $[13,14]$ proposed a model applicable coating cracking that identifies the dimensionless ratio:

$\frac{\Delta H}{H_{\mathrm{o}}} \propto \frac{E_{\mathrm{c}} h^{3}}{d^{3} H_{\mathrm{o}}}$

as being a controlling parameter for describing hardness enhancement. Here $\Delta H$ is the increase in hardness resulting from the presence of the coating, $H_{\mathrm{o}}$ the substrate hardness, $E_{\mathrm{c}}$ the Young's modulus of the coating, $h$ the coating thickness and $d$ is the characteristic diagonal length of indentation.

In the next section we describe the reasoning behind an energy-based model that is suitable for different coating response types (cracking and plasticity), is applicable at a wide range of contact scales, is easy to fit, relies on few empirical fitting parameters, has some basis in physical reality and has the potential to be developed into a predictive design tool.

\subsection{Work of indentation model}

In an indentation experiment with a sharp pyramidal or conical indenter, the highest applied load $P$ is almost invariably found to relate to the maximum penetration depth, $\delta$, (measured after the removal of the load and thus after elastic recovery of any surface flexure but ignoring any small elastic recovery of 
the depth of the indentation itself) by

$P=\frac{H \delta^{2}}{\kappa}$,

where $H$ is the measured hardness and is a parameter describing the indenter geometry. For Vickers indenters $\delta=d / 7$ where $d$ is the indentation diagonal. Similar expressions can be derived for other indenter geometries. The total energy required to produce an indentation of depth $\delta$ is then given by

$W_{\mathrm{tot}}=\int_{\mathrm{o}}^{\delta} P \mathrm{~d} x=\frac{H \delta^{3}}{3 \kappa}$

This is termed the 'work of indentation' and, if measured (as can now readily be achieved with continuous recording indentation techniques (CRIT)) can be used to define an effective value of $H$ which usefully describes the resistance to deformation over the penetration $\delta$. The following derivation of the model will be based on the application of an 'inversion' of this formula, whereby the hardness is expressed in terms of this energy, i.e.

$H=\frac{3 \kappa W_{\text {tot }}}{\delta^{3}}$

Note that no assumptions need to be made about the way the energy was expended. The above expression may therefore be justifiably applied both to the coated system and its substrate separately. It is the comparison between these two situations that will be important.

It is clear that the total energy dissipated in deforming the composite will contain contributions from both the substrate and the coating. However, the partitioning of the energy expended between deformation of the coating and the substrate will vary depending on the scale of indentation. It is convenient to start by considering the case when the indenter penetration depth is very large compared with the coating thickness. Under these conditions the energy term relating to the plastic deformation of the substrate dominates. The energy contribution from the coating is very small in comparison, and one can expect to recover the substrate hardness from Eq. (12). Examining the case for progressively smaller indentation depths, it is clear that while a substrate term (given by Eq. (10)) will remain (but become increasingly smaller), a further term due to the coating will start to become increasingly significant and the energy balance will favour the coating more and more, i.e. the share of its contribution to the total energy will increase.

Total energy expenditure is composed of two parts, the plastic work of deformation in the substrate $\left(W_{\mathrm{s}}\right)$, and deformation and fracture energy in the coating $\left(W_{\mathrm{f}}\right)$, i.e.

$W_{\text {tot }}=W_{\mathrm{s}}+W_{\mathrm{f}}$

Extensive experimental evidence exists on the indentation of hard-coated systems. In developing the present approach, we have reviewed the similarities between deformation and fracture modes observed in a variety of materials used for substrate and coating, and over a range of coating thicknesses and deposition techniques. These observations suggest, that while plastic deformation usually occurs in both coating and substrate, and is primarily responsible for the permanent impression left by the indenter, fracture also occurs at larger loads and indenter displacements (typically, exceeding one tenth of the coating thickness where substrate deformation starts to assume significance [15]). Certain similarities also exist for all hard-coated systems, namely, that coating deformation is most often dominated by cracking, which occurs at the indentation apex, along the edges of the indenter pyramid, and in the form of circumferential cracks around the indentation perimeter. At low loads, fracture is localised along the indenter edges, with possibly one or more peripheral cracks being present. With increasing load, multiple cracking starts to dominate, producing a web pattern concentric around the indenter tip.

In the course of model development, plasticity and fracture must be considered separately, although the resulting models show some convergence, and are combined into a single master formula. This formula was found to be as follows [16]

$H_{\mathrm{c}}=H_{\mathrm{s}}+\frac{H_{\mathrm{f}}-H_{\mathrm{s}}}{1+\left(\beta / \beta_{0}\right)^{X}}$

where the fitting parameters $H_{\mathrm{s}}, H_{\mathrm{f}}-H_{\mathrm{s}}, \beta_{0}$ and $X$ are determined by fitting to the experimentally determined variation of $H_{\mathrm{c}}$ with $\beta$.

\subsection{Experimental validation}

A series of hardness measurements was carried out using conventional Vickers pyramidal testers within the load range approximately $10-200 \mathrm{~N}(1-20 \mathrm{~kg})$, microhardness testing (down to $0.5 \mathrm{~N}$ ) and nanoindentation (down to $5 \mathrm{mN}$ ). The materials studied included: diamond-like carbon coatings, arcdeposited single and multi-layer nitrides and carbo-nitrides of titanium, vanadium, zirconium and niobium, and electroplated and electro-less plated nickel coatings. The substrates used were tool steels and copper [17].

Fig. 1(a) contains hardness plotted against the indenter displacement for sample M053 of McGurk [14] of $2.8 \mu \mathrm{m} \mathrm{NbN}$ on M304. It is clearly unsuitable for interpreting the data and validating any models. Fig. 1(b) re-plots the same hardness data for M053 in partially non-dimensional form. Now, the relative indentation depth $(\beta=\delta / t)$ normalises the indentation depth with respect to the coating thickness, and logarithmic axis is used for this parameter.

It is now apparent from Fig. 1(b) that there is a transition in behaviour which occurs approximately between the points $\beta \sim 1$ and $\beta \sim 0.1$, i.e. for indentation depths between the full coating thickness and down to one tenth of the coating thickness. At depths approximately equal to the coating thickness the hardness reduces asymptotically to the near-substrate value of hardness, whereas for depths shallower than one tenth of the coating thickness the coating hardness "levels off" at a high hardness value, which is likely to be characteristic of the coating itself.

In order to test the capability of the indentation response function, Eq. (14), to describe the full range of hardness variation with indentation depth, a systematic study was undertaken of hardness of nickel coatings of different thicknesses on copper substrates. Copper blanks were used as cathode of a deposition bath with the anode made from $99.9 \%$ commercial purity 

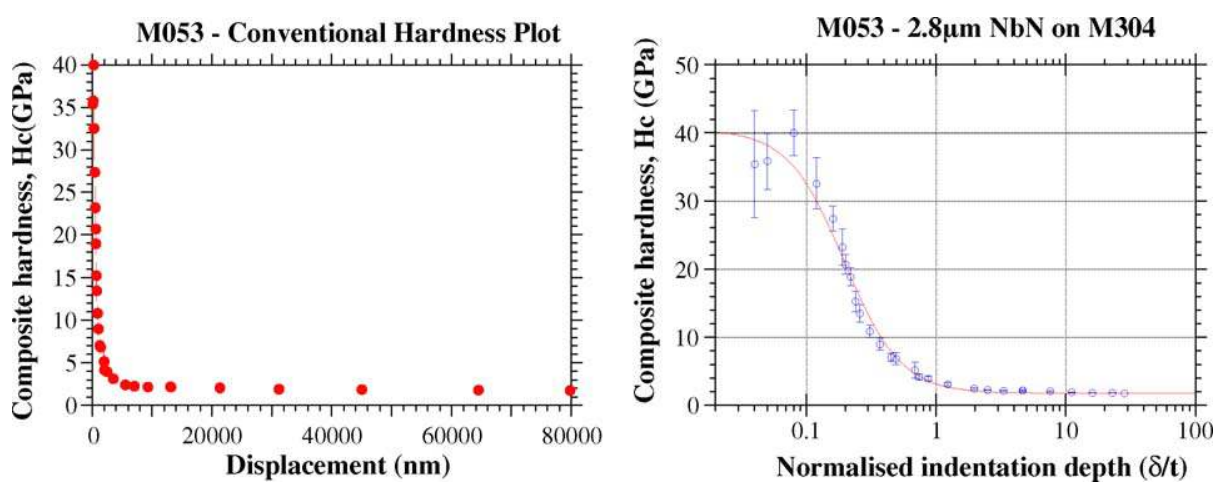

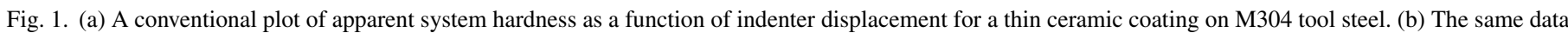

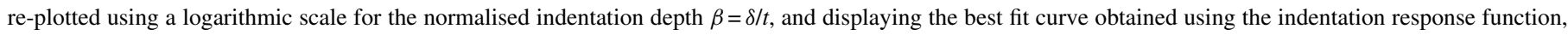
Eq. (14).

nickel. A conventional Watts deposition bath formulation was used, with the solution consisting of $300 \mathrm{~g}$ nickel sulphate, $28 \mathrm{~g}$ of sodium chloride and $40 \mathrm{~g}$ of boric acid, dissolved in $11 \mathrm{of}$ distilled water. The deposition was conducted at a temperature of $55 \pm 1{ }^{\circ} \mathrm{C}$. The current density was regulated using a constant current power supply, so that the thickness of deposition could be accurately controlled for each specimen. The electrodes were mounted a fixed distance apart, and a non-conductive shield was used to improve the directionality of the current flow between them, since the uniformity of the coating thickness depends on the current density distribution.

The coatings were deposited to the nominal thickness of 10 , 20, 30, 40 and $50 \mathrm{~mm}$. The resulting thickness differed slightly from the target values due to the slight variations in the deposition conditions. The actual thickness was measured using ball cratering and metallographic cross sectioning.

The data for each individual coating was first plotted as composite hardness versus indentation depth. The plots clearly possessed a common shape, so in attempt to find a master curve all data was placed on a single graph with the logarithm of relative indentation depth, $\beta$, used as abscissa. The resulting combined plot is shown in Fig. 2. It clearly demonstrates that

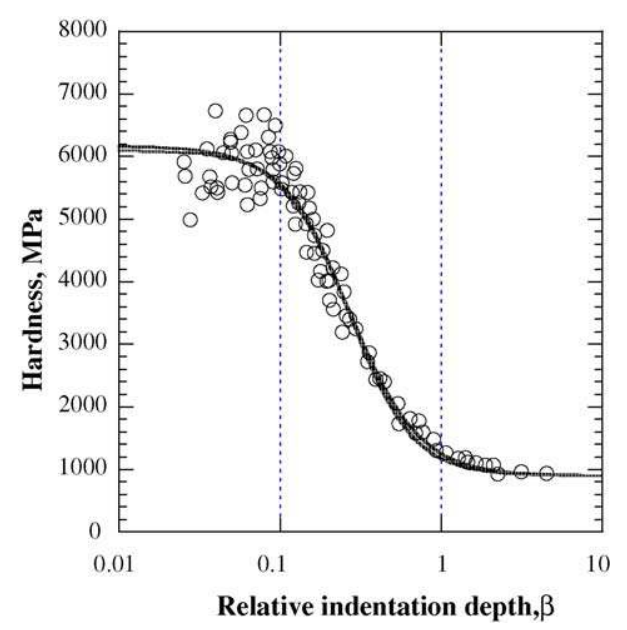

Fig. 2. A combined plot of apparent system hardness vs. the relative indentation depth for a series of Watts electroplated nickel coatings on copper substrates. The coating thickness ranged between 10 and $50 \mu \mathrm{m}$. the overall behaviour of the systems containing coatings of different thickness covering a wide range between 10 and $50 \mu \mathrm{m}$ can nevertheless be very well represented by a single function. The fit curve using indentation response function, Eq. (14), is included in the plot.

\subsection{Finite element simulation}

Finite element analysis was used to gain an insight of the distribution of the mechanical fields in the coated sample during the indentation process. The indenter was considered as a cone with a tip angle of $70.3^{\circ}$ and both the indenter and the layered substrate have been treated as axially symmetric. The choice of this angle is justified by the fact that it gives an equivalent displaced volume equal to that for the Vickers four-sided or Berkovich three-sided pyramid.

The indentation process was simulated using CASTEM2000 FE package, and the analysis was performed under the assumption of large displacements and small rotations coupled with mesh updating after each load increment.

From the point of view of interpretation it is convenient to use the term 'small indentation' to refer to the range of relative indentation depths between zero and unity, and to talk about 'large indentation' for relative indentation depths exceeding unity.

To attain suitable fidelity of FE simulation results at all depths of indentation, from shallow to deep, and to cover at least a decade of scales, three different ranges of relative indentation depth were considered, and different specific meshes were generated for each case ('shallow indentation mesh' for relative indentation depths smaller than $0.1-0.2$, 'medium indentation mesh' for relative indentation depths between $0.1-0.2$ and 0.4-0.5, and 'deep indentation mesh' for relative indentation depth larger than 0.5). Meshes consisted of approximately 4500 linear elements. The choice of the type of element is imposed by the contact modelling routine used by CASTEM2000. It has been shown that this choice does not influence the accuracy of the final results. In order to improve the speed of the computation, an elastic super-element was used to represent the region far removed from the contact zone; the elasticity assumption for this zone was verified a posteriori. The super-element reduced the computational time by about $30 \%$. 


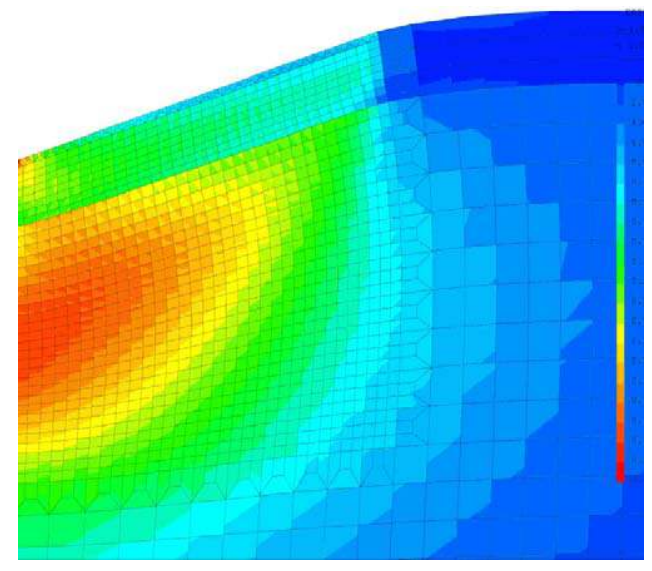

Fig. 3. A map of accumulated plastic strain in the coating and substrate during indentation by an axially symmetric cone punch.

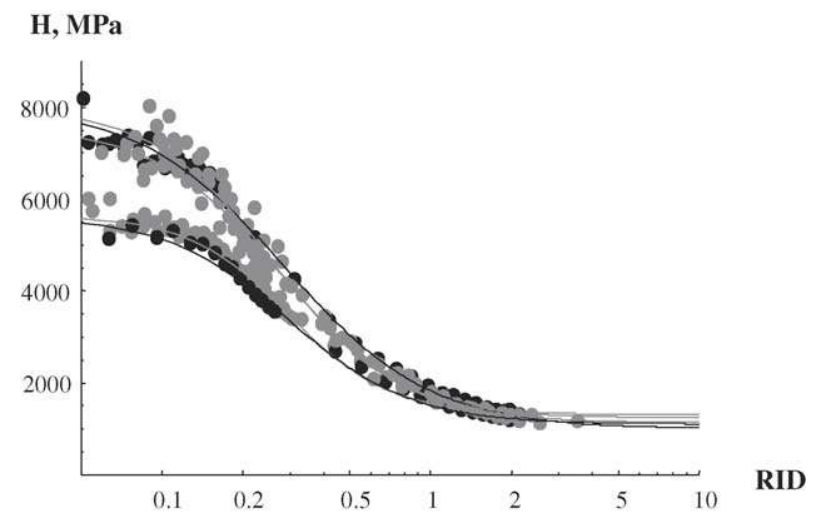

Fig. 4. A comparison between the experimental hardness measurements (grey circles) and the predictions of elasto-plastic finite element analysis (black circles) for Watts electroplated nickel coatings (upper curves) and electro-less nickel coatings (lower curves) on copper substrates.

The indenter was considered as rigid and the layer and the substrate were considered to be elasto-plastic and obey the von Mises yield criterion with non-linear isotropic hardening.

The accumulated plastic strain predicted by the model for the substrate and the coating is illustrated in Fig. 3. A series of finite element calculations was used to determine the variation of the composite hardness as a function of the relative penetration depth, in order to provide a comparison between the predictions of the model illustrated in Fig. 3, and the observed response shown in Fig. 2. The value of apparent hardness was computed from the definition, i.e. by averaging the vertical stress over the entire contact patch, and is shown in Fig. 4.

\section{Contact modulus response}

\subsection{Background}

Similarly to the hardness, the apparent contact stiffness of the coating/substrate combination undergoes a transition between the coating-only value at very low loads and the substrate-dominated value under high loads. Determination of the behaviour at intermediate values of the relative indenta- tion depth $\beta$ requires detailed analysis of the complete problem, taking account of the specific constitutive behaviour of the components. This task of considerable difficulty usually requires the application of laborious numerical methods. It is possible to prove, however, that in the elastic case the solution must depend solely on the combination of the elastic properties of the coating and substrate, the relative indentation depth, and the indenter shape. It then possible to pose the question about finding the transition function which describes the variation with indentation depth of the apparent elastic modulus of the system. This parameter is deduced from the load-displacement curve using the formulae for homogeneous substrate. The solution is required for arbitrary indenter shape, but more specifically we are particularly interested in the important cases of a flat punch, and of conical and parabolic indenter profiles. Analysis can further include the consideration of punch shape imperfections, such as a blunted (rounded) tip of a conical indenter.

The methods of the classical theory of elasticity employed for the solution of elastic contact problems are reviewed in many monographs. The procedure usually involves two essential steps: the derivation of the solution for a concentrated force on the boundary, and inversion of the pair of dual integral equations for the boundary tractions and displacements, written on the basis of this solution.

This approach is employed in the present paper in order to study the dependence of the apparent elastic modulus of a coated system subjected to elastic indentation on the indenter displacement, indenter shape, and the coated system properties. For the purposes of analysis, it is convenient to consider the variation of apparent modulus as a function of the relative indentation depth (contact depth normalised with respect to the coating thickness). The analysis demonstrates that the predicted variation can be very well described by a function belonging to the same family parametric functions introduced for in the previous paragraph. This result allows the response at any load and indenter displacement to be predicted without repeating the calculation for any combination of the layer/substrate properties.

Indenter shape imperfections are well known to impose limitations on the resolution with which the substrate and coating properties can be determined. The present approach allows these limits to be explored quantitatively, with important implications for experimental data collection and interpretation.

The material parameter that directly governs the indention response of elastic homogeneous substrates is referred to as the contact modulus, also known as the plain strain modulus, and is defined as $E^{*}=E /\left(1-v^{2}\right)$. It can be expressed directly as a function of the applied load $P$, the punch shape parameter $f$, the displacement of the indenter $d$, and the contact radius $a_{\mathrm{H}}(d)$ as $E^{*}=P / 2 f d a_{\mathrm{H}}(d)$.

The general solution techniques for frictionless elastic contact mechanics problems for homogeneous substrates were addressed by Sneddon using integral transform methods. The functional dependence of the contact radius $a$ on the indenter displacement for important indenter geometries, such as the cone and sphere, has the form of a power law. This dependence, $a=a_{\mathrm{H}}(d)$, can be used to eliminate the contact radius from the expression for the contact modulus. Consequently, $E^{*}$ can be 
defined in terms of the applied load and indenter displacement. The contact mod is seen to play in the case of the elastic substrates a role similar to that of the hardness in the case of plastic substrates. Thus, for a coated system the apparent contact modulus is defined as:

$E^{*}=\frac{P}{2 f d a_{\mathrm{H}}(d)}$

Coated systems display some important differences from the behaviour of homogeneous substrates. Firstly, for coated systems the apparent contact modulus varies with the indentation displacement $d$. Secondly, the solution for the contact radius as a function of the applied force or indenter displacement is not available for the case of coated systems in closed form, and expressions for the apparent contact modulus cannot be obtained.

\subsection{Integral equation solution}

To analyse a series of indentation problems and to check the validity of the proposed indentation response function, we compute the solutions of elastic indentation problems using a boundary integral approach proposed by Yu et al. [18]. The fundamental advantage of this approach is that both the pressure distribution under the indenter and the displacement profile are assumed to be unknown a priori, but are related to each other through a pair of coupled boundary integral equations. By the use of an elegant transformation, the pair can be turned into a single Fredholm integral equation for a single unknown potential density function. The resulting integral equation is regular, and can be inverted very efficiently using collocation technique.

The numerical implementation of the system of equations described above has been programmed in Mathematica. The solution was found through an iterative procedure. For any chosen contact radius $a$, a Gauss-Legendre quadrature rule was used, and the roots of Legendre polinomials were also employed as collocation points for internal representation of functions and the kernel. This approach allows the boundary integral equation to be transformed into a linear algebraic system and easily solved.

For smooth punches and incomplete contact conditions, the correct value of the contact radius was found by imposing the condition of vanishing contact pressure at the edge of the contact region. Once again, an iterative procedure was used with the dichotomic method, starting with an initial value corresponding to the contact radius in the case of a homogenous substrate.

In order to speed up the numerical computations, one of the memory control options of Mathematica was used (the command form was $f\left[x_{-}\right]:=f[x]=\ldots$.). This avoids repeated evaluation of the same expression, for example, encountered in the integral equation kernel. We also used the automatic compilation procedure in order to speed up the evaluation of functional calls. The accuracy of the solution procedure was tested by comparison with the well-known analytical solutions for homogenous substrates.

Fig. 5(a) illustrates the variation predicted by the model with a conical indenter of the apparent contact modulus for a coated system with the following parameters: $v_{\mathrm{s}}=0.3, v_{\mathrm{f}}=0.2$, $E_{\mathrm{S}}=100 \mathrm{GPa}, E_{\mathrm{f}}=500 \mathrm{GPa}$. It is clear that the indentation response function (continuous lines) provides an accurate representation of the observed variation. At the same time, once the fit parameters are determined, prediction of the apparent contact modulus under any load requires very little calculation compared with the detailed numerical procedure described above.

A further utility of the indentation response function approach is in determining the limits of applicability for various assumptions routinely made during indentation experiments. For example, the tip of a pyramidal (Vickers or Berkovich) or conical indenter is often assumed to be sharp. However, the real observed response may be strongly affected by tip blunting. Interpretation of the results assuming the tip is sharp is likely to lead to significant errors. Using the modelling approach described above we have demonstrated that large inaccuracies arise unless the tip
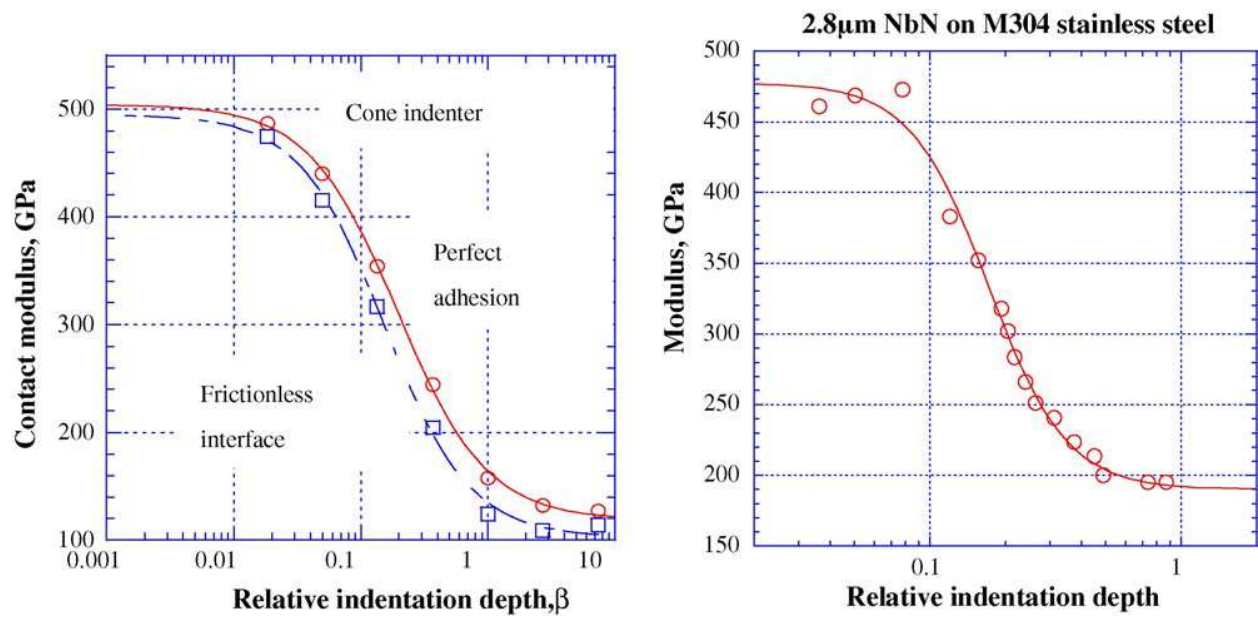

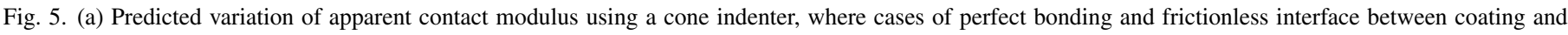

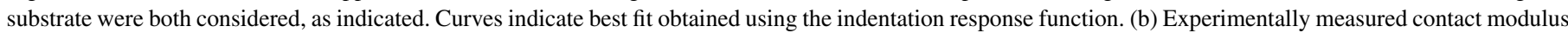

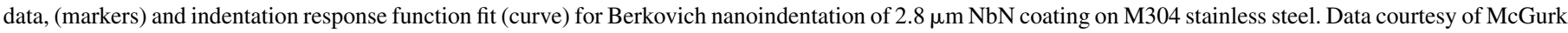
[14]. 
radius is less than about 1/50th of the coating thickness. If this requirement is not observed, the coating stiffness may be overestimated by many tens of percent. This warning is particularly relevant for the cases when the coating is very thin, as is often the case for diamond-like carbon deposits and other films used for hardness and stiffness improvement.

Fig. 5(b) serves as the final illustration of the success of the model in describing the experimentally observed variation of contact modulus. The markers show the data of McGurk [14] for Berkovich nanoindentation of $2.8 \mu \mathrm{m} \mathrm{NbN}$ coating on M304 stainless steel, and the curve shows the model fit to the measurements, exhibiting excellent agreement.

\section{Discussion}

In this paper an overview has been presented of an approach to the property determination of coated systems through the systematic use of the indentation response function.

Both plasticity-dominated and elastic response cases were considered, and experimental data from a very wide range of industrially relevant coated systems was described. In many systems coating cracking was observed. The presence of this deformation mode was found to affect the parameters of the indentation response function, in particular, the exponent $X$ in Eq. (14) [16]. However, even in the presence of cracking the overall response of most systems was still sufficiently well described by the indentation response function. It may be expected, however, that if a drastic change in the deformation mechanism were to occur at a particular load, it will be highlighted by a deviation of the plot from the expected curve.
The presented consideration demonstrates the usefulness of the proposed approach for efficient analysis of coated system properties.

\section{References}

[1] A.M. Korsunsky, M.R. McGurk, S.J. Bull, T.F. Page, Surf. Coat. Technol. 99 (1998) 171-183.

[2] H. Buckle, in: J.H. Westbrook, H. Conrad (Eds.), Science of Hardness Testing and its Research Applications, ASM, Metals Park, Ohio, 1973, p. 453.

[3] B. Jonsson, S. Hogmark, Thin Solid Films 114 (1984) 257.

[4] O. Vingsbo, S. Hogmark, B. Jonsson, A. Ingemarsson, in: P.J. Blau, B.R. Lawn (Eds.), Microindentation Techniques in Materials Science and Engineering, ASTM, Philadelphia, 1986, p. 257.

[5] P.M. Sargent, Ph.D. Thesis, University of Cambridge, 1979.

[6] P.J. Burnett, T.F. Page, J. Mater. Sci. 19 (1984) 845.

[7] P.J. Burnett, D.S. Rickerby, Thin Solid Films 148 (1987) 41

[8] P.J. Burnett, D.S. Rickerby, Thin Solid Films 148 (1987) 51.

[9] S.J. Bull, D.S. Rickerby, Surf. Coat. Technol. 42 (1990) 149.

[10] S.J. Bull, T.F. Page, E.H. Yoffe, Phil. Mag. Lett. 59 (1989) 281

[11] B.R. Lawn, A.G. Evans, D.B. Marshall, J. Am. Ceram. Soc. 63 (1980) 574.

[12] A. Thomas, Surf. Eng. 3 (1987) 117.

[13] M.R. McGurk, H.W. Chandler, P.C. Twigg, T.F. Page, Surf. Coat. Technol. 68/69 (1994) 576.

[14] M.R. McGurk, Ph.D. Thesis, University of Newcastle upon Tyne, 1999.

[15] S.V. Hainsworth, T.F. Page, Mater. Res. Soc. Symp. Proc. 436 (1996) 171.

[16] J.R. Tuck, A.M. Korsunsky, S.J. Bull, R.I. Davidson, Surf. Coat. Technol. 137 (2001) 217.

[17] J.R. Tuck, Ph.D. Thesis, University of Newcastle, Newcastle upon Tyne, England, 2001.

[18] H.Y. Yu, S.C. Sunday, B.B. Rath, J. Mech. Phys. Solids 38 (1990) 745. 